\title{
Metabolic Acidosis Due To Pyroglutamic Acid
}

\author{
Maria João Serpa ${ }^{1}$, Luís Falcão ${ }^{2}$, Susana Franco ${ }^{1}$, Diana Repolho ${ }^{1}$, Nuno Ribeiro Ferreira ${ }^{3}$ \\ ${ }^{1}$ Internal Medicine Department, Hospital Beatriz Ângelo, Loures, Portugal \\ ${ }^{2}$ Nephrology Department, Hospital Beatriz Ângelo, Loures, Portugal \\ ${ }^{3}$ Internal Medicine Department, Centro Hospitalar Lisboa Ocidental, Lisbon, Portugal
}

\section{Received: 15/08/2018}

Accepted: $17 / 09 / 2018$

Published: 27/09/2018

How to cite this article: Serpa MJ, Falcão L, Franco S, Repolho D, Ribeiro Ferreira N. Metabolic acidosis due to pyroglutaminic acid. EJCRIM 2018;5: doi:10.12890/2018_000949.

Conflicts of Interests: The Authors declare that there are no competing interests.

This article is licensed under a Commons Attribution Non-Commercial 4.0 License

\section{ABSTRACT}

Metabolic acidosis is a common clinical disturbance due to increased plasma acidity caused by a primary decrease in serum HCO3concentration. It is classified as normal or high anion gap metabolic acidosis. High anion gap metabolic acidosis can result from either a decrease in unmeasured cations $\left(\mathrm{K}^{+}, \mathrm{Ca}^{+}, \mathrm{Mg} 2^{+}\right)$or an increase in unmeasured anions ( $\mathrm{PO}_{4}{ }^{3-}$, albumin). However, other anions such as lactic acid or keto acids may cause this acid-base disorder. It can also result from renal failure and intoxication (salicylate, methanol, ethylene glycol), or more rarely, from massive rhabdomyolysis and pyroglutamic (5-oxoproline) acidemia. Acidemia due to pyroglutamic acid should be considered when no other aetiology is found. High anion gap metabolic acidosis is diagnosed in children with inherited defects in enzymes of the $y$-glutamyl cycle. In adults, this disorder from pyroglutamic acid has been described in association with chronic acetaminophen misuse. We report a case of pyroglutamic acidosis in a woman with acute misuse of acetaminophen concurrent with chronic use.

\section{LEARNING POINT}

- Paracetamol is an easily available drug with potentially harmful consequences.

- Accumulation of pyroglutamic acid (5-oxoproline) may be a cause of high anion gap metabolic acidosis.

- Reporting cases of 5-oxoprolinemia acidosis contributes to better understanding of the condition.

\section{KEYWORDS}

High anion gap metabolic acidosis, 5-oxoprolinemia, pyroglutamic acidemia, acetaminophen, paracetamol

\section{CASE DESCRIPTION}

A 55-year-old woman was brought to the emergency department after ingestion of more than $10 \mathrm{~g}$ of acetaminophen the same day, with little relief of pain. The patient had a 12-year history of breast cancer treated with chemotherapy and radiotherapy, with chronic pain from bone metastasis diagnosed the year previously (rib cage, sacroiliac joint and pubic bone). Her medications included hormones, opioids and non-prescribed analgesics.

On examination, the patient was alert, her blood pressure was $156 / 80 \mathrm{mmHg}$, her temperature was $36^{\circ} \mathrm{C}$, and she was tachycardic $(120$ beats per minute) and short of breath (respiratory rate of 40 per minute, with $98 \%$ oxygen saturation on room air). The remaining physical examination was unremarkable except for cachexia.

The results of her initial laboratory evaluation were: severe metabolic acidosis (pH 7.15, pCO2 $10 \mathrm{mmHg}, \mathrm{pO} 2112 \mathrm{mmHg}$, bicarbonate $3.5 \mathrm{mmol} / \mathrm{l}$, lactate $7 \mathrm{mg} / \mathrm{dl}$ (reference range $4.5-18 \mathrm{mg} / \mathrm{dl}$ )) in arterial blood gas; creatinine $2.15 \mathrm{mg} / \mathrm{dl}$, sodium $138 \mathrm{mmol} / \mathrm{l}$, potassium 5.2 
mmol/l, chloride 104 mmol/l, ionized calcium $8.2 \mathrm{mg} / \mathrm{dl}$, phosphate $2.6 \mathrm{mg} / \mathrm{dl}$, magnesium $2.1 \mathrm{mg} / \mathrm{dl}$, albumin $3 \mathrm{mg} / \mathrm{dl}$, anion gap 40 mmol/l; aspartate aminotransferase $66 \mathrm{U} / \mathrm{l}$, alanine aminotransferase $69 \mathrm{U} / \mathrm{l}$, alkaline phosphatase $120 \mathrm{U} / \mathrm{l}$, Y-glutamyl transferase $160 \mathrm{U} / \mathrm{l}$ and total bilirubin $0.19 \mathrm{mg} / \mathrm{dl}$, with no coagulation abnormalities. Lactate dehydrogenase was $356 \mathrm{U} / \mathrm{l}$ and CK was normal (rhabdomyolysis was excluded). The acetaminophen concentration was $103.4 \mathrm{mg} / \mathrm{l}$. Ethanol, salicylate and beta-hydroxybutyrate levels were undetectable. The $\mathrm{N}$-acetylcysteine protocol was initiated as well as intravenous fluids with sodium bicarbonate.

Two hours after admission and initial treatment, the patient deteriorated with worsened Kussmaul breathing, a respiratory rate of 44 per minute, arterial blood gas with decreased bicarbonate $(3 \mathrm{mmol} / \mathrm{l})$ and pCO2 $9 \mathrm{mmHg}$, lactate $13 \mathrm{mg} / \mathrm{dl}$ despite medical treatment. The patient required mechanical ventilation and was admitted to the ICU. She was started on hydration, bicarbonate and $\mathrm{N}$-acetylcysteine. The patient improved over the following days and was extubated. Her transaminase levels and renal function had returned to normal by the third day, which obviated the need for dialysis. Her $\mathrm{pH}$ normalized, the anion gap reduced to $13 \mathrm{mmol} / \mathrm{l}$ and the acetaminophen level decreased to $37 \mathrm{mg} / \mathrm{l}$.

The diagnosis of high anion gap metabolic acidosis secondary to 5-oxoprolinemia (pyroglutamic acidemia) was considered due to the lack of any other unifying diagnosis. An anion gap of $40 \mathrm{mmol} / \mathrm{I}$ clearly indicates the presence of an organic acid, while the absence of lactic, keto and other acids suggested accumulation of 5-oxoproline as an explanation for the severe acidosis.

\section{DISCUSSION}

Acetaminophen misuse documented by clinical history, a lack of other causes of severe metabolic acidosis and a good response to treatment should raise clinical suspicion of 5-oxoproline (pyroglutamic acid) accumulation ${ }^{[1,2]}$. In our patient, anamnesis excluded the most common causes of metabolic acidosis, such as propylene and methanol poisoning, hyperlactatemia, salicylate abuse, renal disease and ketoacidosis (GOLD MARK mnemonic) ${ }^{[2,3]}$. Our diagnosis was therefore one of exclusion, because none of the other causes fit our patient, and was corroborated by the good clinical evolution with treatment.

Urine or serum measurements of pyroglutamic acid (5-oxoproline) are performed only in specialist laboratories ${ }^{[2,4]}$ and are not readily available for every doctor. However, the diagnosis can be made using tools accessible in all hospitals.

In 5-oxoproline metabolic acidosis, acetaminophen increases use of glutathione and promotes depletion of glutathione storage ${ }^{[2,5]}$. However, normal cells are able to increase glutathione production when stressed with acetaminophen, so it is only with high acetaminophen concentrations that glutathione stores are seriously depleted and substantial cellular toxicity occurs ${ }^{[3]}$. About $10 \mathrm{~g}$ of paracetamol are required before the availability of glutathione is overwhelmed, although only $4 \mathrm{~g}$ are needed in undernourished patients.

The epidemiology is unknown due to the large number of undiagnosed cases, but most patients, as was ours, are malnourished women ${ }^{[1-4]}$ and the condition is exacerbated by renal insufficiency ${ }^{[1,2,4]}$. Chronic paracetamol abuse is described in the literature, although in our patient, intoxication occurred as a result of extra paracetamol ingestion in addition to her usual daily dose.

Treatment is mainly supportive, with paracetamol withdrawal being key in chronic cases ${ }^{[2]}$. Supportive treatment consists of fluid repletion, and bicarbonate and $\mathrm{N}$-acetylcysteine administration ${ }^{[2]}$. $\mathrm{N}$-acetylcysteine use is widely used ${ }^{[5]}$ despite few supportive studies, as it has a theoretical benefit and few adverse effects. In acetaminophen intoxication, $\mathrm{N}$-acetylcysteine restores the hepatic levels of glutathione ${ }^{[5]}$ and increases the negative feedback of $y$-glutamylcysteine synthetase, resulting in reduced generation of pyroglutamic acid from $\mathrm{Y}$-glutamylcysteine. $\mathrm{N}$-acetylcysteine increases and replenishes glutathione storage by acting as a precursor of glutathione synthesis ${ }^{[2,5]}$ and also has an antioxidant effect. Any underlying conditions (such as infection) must be treated ${ }^{[2]}$. In refractory cases, haemodialysis may be needed to remove acetaminophen from the blood.

As acetaminophen is a commonly used over-the-counter medication, which is widely prescribed often in maximal doses, given its innocuous reputation ${ }^{[4]}$, recognition of this rare complication of acetaminophen (paracetamol) poisoning is paramount.

\section{REFERENCES}

1. Vichot A, Rastegar A. Use of anion gap in the evaluation of a patient with metabolic acidosis. Am J Kidney Dis 2014;64:653-657.

2. Abkur T, Mohammed W, Ali M, Casserly L. Acetaminophen-induced anion gap metabolic acidosis secondary to 5-oxoproline: a case report. J Med Case Rep 2014;8:409.

3. Duewall J, Fenves A, Richey D, Tran L, Emmett M. 5-Oxoproline (pyroglutamic) acidosis associated with chronic acetaminophen use. Proc (Bayl Univ Med Cent) 2010;23:19-20.

4. Howie S, Tarn A, Soper C. A not so simple analgesic. NDT Plus 2010;3:573-575.

5. Lawrence D, Bechtel L, Charlton N, Holstege C. 5-Oxoproline-induced anion gap metabolic acidosis after an acute acetaminophen overdose. J Am Osteopath Assoc 2010;110:545-551. 